\title{
Article \\ Effect of Liquid Grounding Electrode on the NOx Removal by Dielectric Barrier Discharge Non-Thermal Plasma
}

\author{
Xiu Xiao ${ }^{1}$, Yu Guo ${ }^{1}$, Zongyu Wang ${ }^{1, *}\left(\mathbb{C}\right.$, Wei Zhang ${ }^{1}$, Jifeng Zhang ${ }^{1,2}$, Yulong Ji ${ }^{1}{ }^{\circledR}$ and Chao Chang ${ }^{1}$ \\ 1 College of Marine Engineering, Dalian Maritime University, Dalian 116026, China; \\ xiaoxiu@dlmu.edu.cn (X.X.); guoyu0411@163.com (Y.G.); zhangwei090530@126.com (W.Z.); \\ zhangjifeng@dlmu.edu.cn (J.Z.); jiyulong@dlmu.edu.cn (Y.J.); chang3223426@126.com (C.C.) \\ 2 Yangtze Delta Region Institute of Tsinghua University, Jiaxing 314006, China \\ * Correspondence: wangzongyu09@163.com
}

Citation: Xiao, X.; Guo, Y.; Wang, Z.; Zhang, W.; Zhang, J.; Ji, Y.; Chang, C. Effect of Liquid Grounding Electrode on the NOx Removal by Dielectric Barrier Discharge Non-Thermal Plasma. Appl. Sci. 2021, 11, 8815. https://doi.org/10.3390/ app11198815

Academic Editor: Cristian FOCSA

Received: 15 August 2021

Accepted: 15 September 2021

Published: 22 September 2021

Publisher's Note: MDPI stays neutral with regard to jurisdictional claims in published maps and institutional affiliations.

Copyright: (c) 2021 by the authors. Licensee MDPI, Basel, Switzerland. This article is an open access article distributed under the terms and conditions of the Creative Commons Attribution (CC BY) license (https:// creativecommons.org/licenses/by/ $4.0 /)$.

\begin{abstract}
In this paper, an experimental setup was established to study the influence of potassium chloride (KCL) solution as the ground electrode on the nitrogen oxides (NOx) removal efficiency in non-thermal plasma (NTP) generated by dielectric barrier discharging (DBD) reactor. The experimental results show that the KCL solution as the ground electrode has better stability and higher discharge intensity and it is a promising approach to improve NOx removal efficiency. The specific NOx removal efficiency is related to the power frequency, the concentration and temperature of the KCL solution. As the power frequency increases, the NOx removal efficiency first increases and then decreases, and a maximum value is reached at the power frequency of $8 \mathrm{kHz}$. The NO removal effect is improved as the concentration of the KCL solution increases, especially when the concentration is lower than $0.1 \mathrm{~mol} / \mathrm{L}$. Under the same KCL solution concentration and input energy density, the NOx removal efficiency is increased with the solution temperature. In particular, when the power discharge frequency is $8 \mathrm{kHz}$, the KCL solution concentration is $0.1 \mathrm{~mol} / \mathrm{L}$ and the solution temperature is $60^{\circ} \mathrm{C}$, the NOx and NO removal efficiency reach $85.82 \%$ and $100 \%$, respectively.
\end{abstract}

Keywords: liquid ground electrode; NOx removal; non-thermal plasma; DBD reactor; KCL solution

\section{Introduction}

Nitrogen oxides (NOx), as one of the main air pollutants, is mainly produced from the combustion of fossil fuels in human activities, of which industrial emissions and transportation emissions account for the largest proportion [1]. Large amounts of NOx emissions will cause acid deposition, deterioration of water quality, and damage to the earth's vegetation and ecological environment, severely endangering industrial and agricultural production. In addition, $\mathrm{NOx}$ has a synergistic effect on the concentration of fine particulate matter (PM2.5) and ozone $\left(\mathrm{O}_{3}\right)$, and is one of the inducing factors of human respiratory and cardiovascular diseases [2]. NOx is easy to decompose under light and reacts chemically with other hydrocarbon compounds in the air to form more toxic photochemical smog, causing greater pollution [3]. In order to reduce the harm of NOx to the environment, a lot of NOx removal researches have been conducted and many solutions have been proposed. Among them, exhaust gas recirculation (EGR) and selective catalytic reduction (SCR) are both relatively efficient treatment technologies [4]. Recently, the non-thermal plasma (NTP) NOx removal technology has attracted wide attention for its advantages of and compact system structure, and significant progress has been made [5-7]. At present, the NTP NOx removal technologies mainly include corona discharge and dielectric barrier discharge (DBD) plasma. DBD technology has been widely studied because of its strong discharge stability, high energy utilization efficiency, and better prospects in industrial applications [8].

DBD discharge is to insert insulating medium between two electrodes while applying high-voltage alternating current. The insertion of the double insulating medium can 
prevent the electrode from directly contacting the discharge gas, avoid electrode corrosion, and prolong the service life of the equipment [9]. Compared to other NTP technologies, DBD discharge is more uniform, which can improve the discharge intensity and increase the range of discharge corona [10]. Moreover, research has shown that DBD has a higher NO removal efficiency under a lower energy density and a shorter residence time [11]. However, the NOx removal efficiency of the DBD plasma technology is affected by many factors. Chen [12] studied the structural characteristics of the DBD reactor, and the results showed that the overall removal effect of the coaxial cylindrical reactor was better than that of the flat plate, and the glass medium material had a better effect than mica. Bratislav [13] studied the effects of direct oxidation and indirect oxidation on the NOx removal efficiency of DBD plasma. The NO removal efficiency of direct oxidation was only $30 \%$, while for indirect oxidation it reached $70 \%$. Yoon [14] investigated the effect of catalyst on $\mathrm{NO}$ removal efficiency by coupling the catalyst $\gamma-\mathrm{Al}_{2} \mathrm{O}_{3}$ and basic zeolite $\mathrm{NaY}$ with the DBD plasma. When the catalyst was NaY, the NO removal efficiency was $50 \%$ at the gas temperature of $180-230^{\circ} \mathrm{C}$. After coupling with $\gamma-\mathrm{Al}_{2} \mathrm{O}_{3}$, the $\mathrm{NO}$ removal efficiency reached $80 \%$ at the temperature of $340{ }^{\circ} \mathrm{C}$. Cao [15] designed line-plate and parallel-plate dielectric barrier discharge reactors for NOx removal, and the final NO removal efficiency reached $60 \%$. Yang [16] combined DBD with negative pulse corona to remove NO. When the $\mathrm{O}_{2}$ concentration was $3 \%$ and $0.5 \mathrm{vol} \%$ ethanol was added, the NO removal efficiency reached $95 \%$. Wang [17] studied the effects of different electrodes such as plate electrodes, mesh electrodes, and water electrodes on the removal efficiency of NOx. The results showed that the discharges of the three-electrode materials were all filamentary discharges, and the discharge effect of pure water was the weakest.

It can be indicated from the above researches that the discharge effect of the DBD and the removal efficiency of NOx are severely affected by the structure of the reactor, the dielectric material, and the electrode material. Significantly, existing researches on the removal of NOx by DBD plasma have mostly focused on the DBD exciters of the surface type and parallel-plate configuration, and the coaxial cylindrical DBD reactor ground electrode is usually a metal mesh [18]. The discharge of the metal mesh electrode is a point discharge and the discharge effect is greatly affected by the number of meshes. Moreover, the metal mesh electrode is prone to be not compact when it is arranged. In response to these problems, this paper proposes a DBD reactor using a coaxial cylindrical stainlesssteel rod as the high-voltage electrode and KCL solution as the ground electrode for NOx removal. Compared with the water electrode mentioned by Wang [17], the conductivity of the KCL solution is higher, which is expected to make the discharge of the DBD reactor more uniform and more effective. Meanwhile, the influence of the temperature rising effect of the DBD reactor discharge on the experimental results can be reduced, which is conducive to basic research.

\section{Experimental}

\subsection{Experimental System}

An experimental setup was established to study the influence of KCL solution as the ground electrode on the NOx removal of dielectric barrier discharge non-thermal plasma. As shown in Figure 1, the experimental system mainly includes a gas distribution system, a plasma power supply device, a DBD reactor, a liquid electrode circulation system, and a flue gas analyzer (Testo350, Freiburg, Germany), etc. The gas distribution system is composed of several high-pressure gas cylinders, pressure reducing valves, and mass flow controllers (Beijing Sevenstar CS200AD, Beijing, China). For more details of the gas distribution system, please refer to Reference [19]. The $\mathrm{O}_{2}$ used in the experiment is highpurity standard gas, and the $\mathrm{NO} / \mathrm{NO}_{2}$ used $\mathrm{N}_{2}$ as the standard carrier gas (Dalian Date Gas Co., Ltd., Dalian, China). The proportion of each gas component is adjusted by the corresponding mass flow controller. During the experiment, each component gas is output from the high-pressure gas cylinder, mixed evenly in the pipeline at the front end of the DBD reactor after passing through a flow meter, and then enters the DBD plasma reactor for 
reaction. The plasma power supply uses a high-voltage alternating current (Nanjing Suman CTP-2000K, Nanjing, China), and a digital oscilloscope (Agilent MSO7104B, PaloAlto, Santa Clara, CA, USA) is applied to obtain the electrical characteristics. In order to analyze the gas composition after the reaction, a flue gas analyzer is used to detect the volume concentration of $\mathrm{NO}$ and $\mathrm{NO}_{2}$ in the reaction gas, and an emission spectrometer (AvaSpec-ULS2048CL, Apeldoom, The Netherlands) is adopted to analyze the spectrum released from the plasma discharge region.

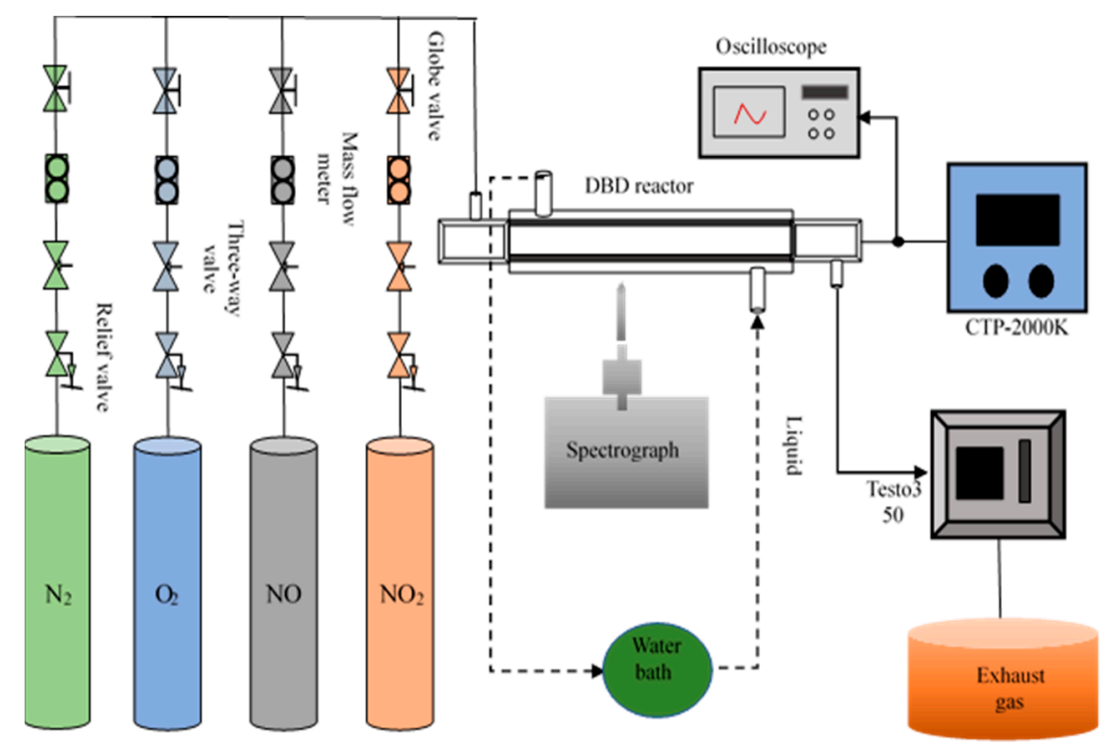

Figure 1. Schematic diagram of the experimental system.

\subsection{DBD Reactor}

The structure of the DBD plasma reactor is a traditional coaxial cylinder, as displayed in Figure 2. The high-voltage electrode is a solid stainless-steel rod with a diameter of $24 \mathrm{~mm}$ and an actual reactor length of $250 \mathrm{~mm}$, and the discharge gap is $2 \mathrm{~mm}$. A quartz glass tube with an outer diameter of $26 \mathrm{~mm}$ and an inner diameter of $24 \mathrm{~mm}$ is used as the barrier medium, and the external circulating liquid is applied as the ground electrode. The gas inlet and outlet are respectively located on the fastening sleeves on the left and right sides of the reactor, and both have a diameter of $7 \mathrm{~mm}$.

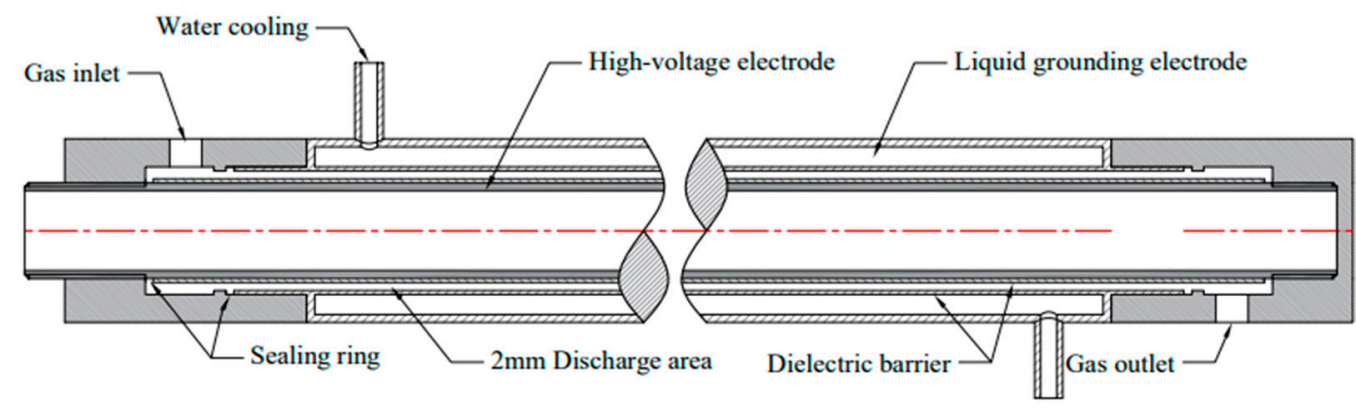

Figure 2. Structure diagram of the plasma reactor.

\subsection{Ground Electrode}

The distinguished characteristic of this study lies in the application of KCL solution as the ground electrode. The purity of KCL reagent (Xilong Science Co., Ltd., Xi'an, China) is greater than $99.5 \%$. The KCL solution was prepared with distilled water as the solvent. During the experiment, the conductivity of the KCL solution with different concentrations was measured by a conductivity meter (Yueping DDS-11A, Shanghai, China) in real-time, and the values are shown in Table 1. 
Table 1. Electrical conductivity of the KCL solution at different concentrations $\left(20^{\circ} \mathrm{C}\right)$.

\begin{tabular}{cc}
\hline KCL Solution Concentration (mol/L) & Electrical Conductivity $(\mathbf{m s} / \mathbf{c m})$ \\
\hline 0.001 & 0.25 \\
0.01 & 1.37 \\
0.1 & 10.00 \\
1.0 & 72.80 \\
\hline
\end{tabular}

\subsection{Data Analysis}

The initial gas compositions of the NOx removal experiment were as follows: $\mathrm{O}_{2}(5 \%)$, $\mathrm{NO}$ (500 ppm), and $\mathrm{NO}_{2}(50 \mathrm{ppm}) . \mathrm{N}_{2}$ was used as the carrier gas and the total gas flow rate was $2 \mathrm{~L} / \mathrm{min}$. The energy density of the DBD reactor was determined by the input power, and the calculation formula is:

$$
S E D=\frac{60 \times P_{\text {in }}}{1000 \times Q_{\text {Exhaust }}}
$$

where $S E D$ is the energy density of the DBD reactor $(\mathrm{kJ} / \mathrm{L}) ; P_{\text {in }}$ is the power delivered by the plasma power supply $(\mathrm{W})$, and $Q_{\text {Exhaust }}$ is the flow rate of the experimental gas $(\mathrm{L} / \mathrm{min})$.

The calculation method of nitrogen oxide removal efficiency is as follows:

$$
\eta=\frac{C_{1}\left(N O_{2}+N O\right)-C_{2}\left(N O_{2}+N O\right)}{C_{1}\left(N O_{2}+N O\right)} \times 100 \%
$$

where $\eta$ is the nitrogen oxide removal rate (\%), $C_{1}$ is the initial nitrogen oxide concentration $(\mathrm{ppm})$, and $C_{2}$ is the nitrogen oxide concentration at the end of the reaction (ppm).

\section{Results and Discussion}

\subsection{Electrical Parameter Characteristics of the DBD Reactor}

As can be seen in the previous literature [20], the volt-ampere characteristic curves of the coaxial cylindrical DBD reactor using copper mesh as the ground electrode have many protrusions, which indicated that the discharge is unstable and the beating amplitude is large. When KCL solution was introduced as the ground electrode in the present study, the electrical characteristic curves of the coaxial cylindrical DBD reactor are shown in Figure 3, where $U_{\text {out }}(V)$ and $U_{m}(V)$ represent the output voltage and the capacitor voltage after sampling the current, respectively. The detailed calculation process of the current can be found in Reference [19]. The current flowing through the loop varies with $U_{m}$. The smoothness of the capacitor voltage curve can determine whether the current is stable. Obviously, the volt-ampere characteristic curves obtained in this experiment are much smoother without significant protrusions, demonstrating that the discharge stability of the plasma reactor is stronger, and the discharge is more uniform and diffuse comparing with the results of Reference [19]. The reason for this phenomenon is that the discharge channel formed in the DBD reactor is more stable when liquid is used as the ground electrode. In contrast, the metal mesh ground electrode will change the discharge channel due to the ionization corrosion of the electrode, resulting in unstable discharge.

\subsection{The Influence of Power Frequency on NOx Removal Efficiency}

According to the ionization mechanism of Townsend, when a high voltage alternating current is applied between two electrodes, the strong electric field will accelerate the collision between the electrons and the neutral gas particles near the electrodes [21]. The variation of the power supply frequency will change the collision frequency (referring to the non-isotropic collision frequency affected by the applied electric field) and the free stroke of the electrons. Specifically, when the power frequency is very low, the collision frequency of the high-energy electrons with the neutral particles is too low for the electrons to be ionized. When the frequency is too high, the free path of the electrons is shortened and the energy obtained by the electrons is less, which will also increase the difficulty 
of ionization. Therefore, a discharge frequency test was performed on the DBD reactor before the experiment. The results showed that the plasma reactor did not discharge when the power supply frequency was lower than $6 \mathrm{kHz}$. Since in DBD discharge, subsequent discharges are more likely to occur than initial discharges due to wall charges (residual charges) attached to the dielectric barrier. Therefore, in the subsequent experiments, the measured voltages are all continuous discharge voltages, which are the plasma driving voltages. However, when the power frequency exceeded $10 \mathrm{kHz}$, the sustaining voltage at the start of discharge was so high that the subsequent experimental voltage could easily overtake the maximum value of the plasma power supply. Based on this phenomenon, four power frequencies of $7 \mathrm{kHz}, 8 \mathrm{kHz}, 9 \mathrm{kHz}$, and $10 \mathrm{kHz}$ were selected for the follow-up experiments. As displayed in Figure 4, the sustaining voltage of the first discharge in each case is the very first point of each curve in Figure 4: the sustaining voltage during the first discharge is $30 \mathrm{~V}$ at $7 \mathrm{kHz}, 50 \mathrm{~V}$ at $8 \mathrm{kHz}, 110 \mathrm{~V}$ at $9 \mathrm{kHz}$, and $220 \mathrm{~V}$ at $10 \mathrm{kHz}$. Moreover, as the frequency increases, the sustaining voltage at which discharge starts is greater. Furthermore, under the same power frequency, the energy density (SED) increased almost linearly as the voltage increased.

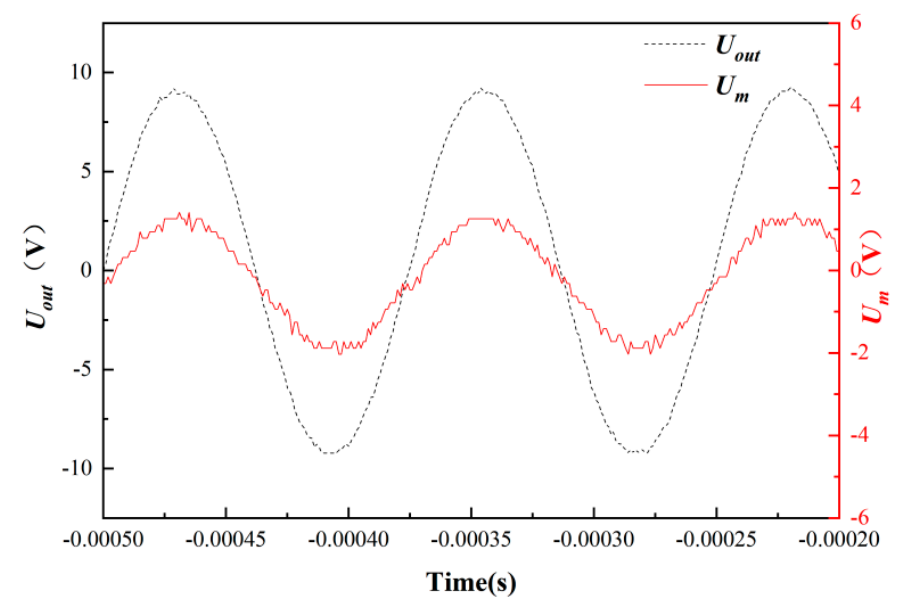

Figure 3. DBD plasma reactor electrical parameter curve.

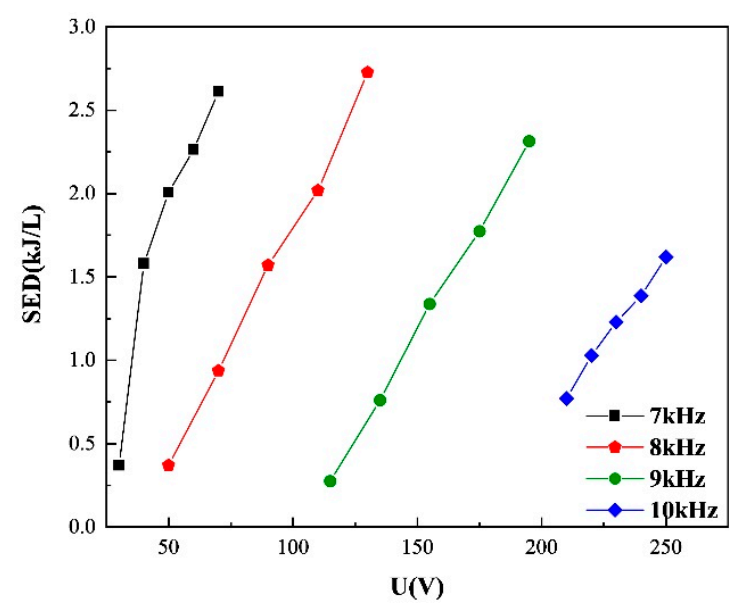

Figure 4. The variation of DBD discharge density with discharge voltage under different discharge frequencies.

The influence of power frequency on NO removal efficiency is exhibited in Figure 5a, where the concentration of KCL solution is $1.0 \mathrm{~mol} / \mathrm{L}$. It can be seen that when the discharge frequency is lower than $10 \mathrm{kHz}$, the final NO removal efficiency can reach $100 \%$. Moreover, the NO removal efficiency increases with the energy density. When the input energy 
density is the same, the overall $\mathrm{NO}$ removal efficiency is the best at the discharge frequency of $8 \mathrm{kHz}$. Figure $5 \mathrm{~b}$ shows the profiles of the variation of NOx removal efficiency with power frequency and energy density. On the one hand, the NOx removal efficiency at each power frequency increases continuously with the energy density. On the other hand, when the energy density is kept constant, the NOx removal efficiency presents a characteristic of first increasing and then decreasing as the power frequency increases. This can be explained by the fact that at the discharge frequency of $7 \mathrm{kHz}$, the collision frequency of high-energy electrons and neutral particles is very low, which makes it difficult for the gas molecules to ionize. Therefore, the reaction rate of the main reaction occurring in the DBD reactor is slower than that when the discharge frequency is $8 \mathrm{kHz}$, and the removal efficiency is lower.
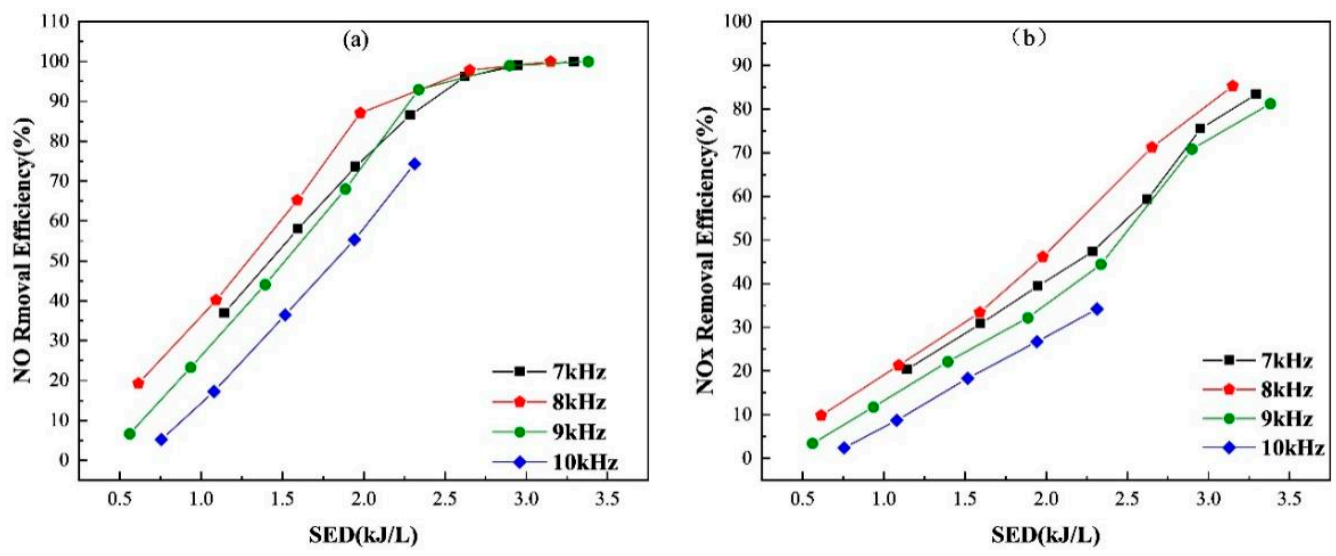

Figure 5. The influence of discharge frequency on NOx removal efficiency at different SEDs: (a) NO removal efficiency; (b) NOx removal efficiency.

When the discharge frequency exceeds $8 \mathrm{kHz}$, as the frequency increases, a larger discharge power and energy density are required to obtain the same NOx removal efficiency, which will cause the temperature of the discharge area to rise. The main chemical reactions for NOx removal in the DBD reactor are shown in Formulas (3)-(10), where the reaction rate of Reaction (9) is much smaller than that of Reaction (10). The increase in power frequency will increase the collision rate of active particles with $\mathrm{NO}$, thereby accelerating the reaction rate of Reactions (7) and (9). However, the Reaction (3) is an exothermic reaction and the rise of temperature in the discharge region will shift the balance of the reversible reaction to the left [22], so that there is not enough $\mathrm{O}_{3}$ for Reaction (10). The discharge frequency is not the only factor that affects the NOx removal efficiency. In this case, as the power frequency increases, more energy is consumed on the high-voltage electrode of the DBD. As a result, the reactor temperature is increased and Reaction (3) accelerates to the left, so that the reaction rate of Reaction (10) as well as the NOx removal efficiency are reduced [23]. Therefore, when the discharge frequency is greater than $8 \mathrm{kHz}$, the NOx removal efficiency is reduced as the discharge frequency increases. Since the maximum regulated voltage of the plasma power supply is $250 \mathrm{~V}$, the reaction is ended with a NOx removal efficiency of $34.19 \%$ when the energy density reaches $2.313 \mathrm{~kJ} / \mathrm{L}$ at the power frequency of $10 \mathrm{kHz}$. Overall, under the same energy density, the NOx removal effect is the best at the power supply frequency of $8 \mathrm{kHz}$. In particular, when the energy density is $3.105 \mathrm{~kJ} / \mathrm{L}$, the NOx removal efficiency reaches a maximum of $85.24 \%$.

$$
\begin{gathered}
3 \mathrm{O}_{2} \rightleftharpoons 2 \mathrm{O}_{3} \\
e+\mathrm{N}_{2} \rightarrow e+\bullet \mathrm{N}+\bullet \mathrm{N} \\
e+\mathrm{O}_{2} \rightarrow e+\bullet \mathrm{O}+\bullet \mathrm{O} \\
\bullet \mathrm{N}+\bullet \mathrm{O} \rightarrow \mathrm{NO}
\end{gathered}
$$




$$
\begin{gathered}
\bullet \mathrm{N}+\mathrm{NO} \rightarrow \mathrm{N}_{2}+\bullet \mathrm{O} \\
\bullet \mathrm{O}+\mathrm{O}_{2} \rightarrow \mathrm{O}_{3} \\
\bullet \mathrm{O}+\mathrm{NO} \rightarrow \mathrm{NO}_{2} \\
\mathrm{O}_{3}+\mathrm{NO} \rightarrow \mathrm{NO}_{2}+\mathrm{O}_{2}
\end{gathered}
$$

\subsection{The Influence of Solution Concentration on NOx Removal Efficiency}

As can be seen from Table 1, the concentration of KCL solution affects its conductivity significantly. Therefore, when KCL solution is used as the ground electrode, the solution concentration is one of the important factors affecting the NOx removal effect. The variation of energy density with input voltage at different solution concentrations is shown in Figure 6 . When the solution concentration is $0.001 \mathrm{~mol} / \mathrm{L}$, the sustaining voltage at the start of discharge is about $60 \mathrm{~V}$, which is $10 \mathrm{~V}$ higher than the sustaining voltage at the start of discharge corresponding to other solution concentrations. In addition, the energy density under each input voltage is the largest at such concentration, indicating that the discharge in the DBD reactor requires greater energy consumption. As the concentration of the solution increases, the energy density corresponding to the same voltage becomes smaller. Interestingly, when the solution concentration is $0.1 \mathrm{~mol} / \mathrm{L}$ and $1.0 \mathrm{~mol} / \mathrm{L}$, respectively, the profiles of the energy density versus input voltage basically coincide. That is, after the conductivity reaches a certain level, it has little influence on the discharge effect of the DBD reactor.

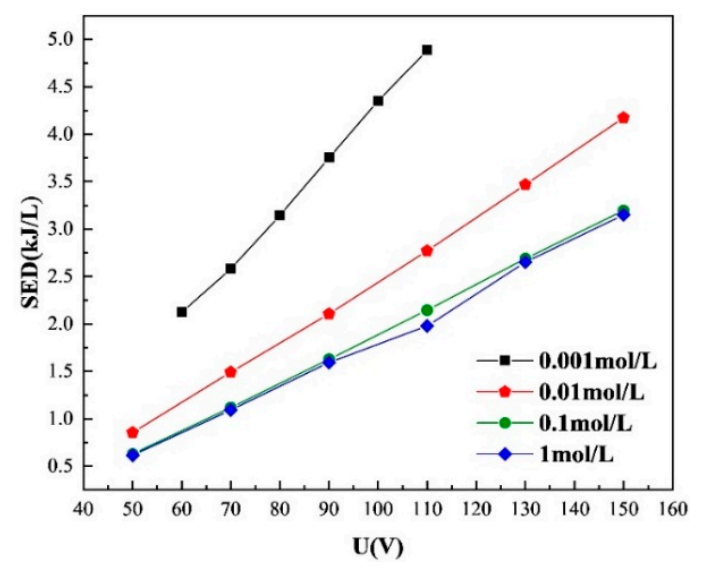

Figure 6. The variation of energy density with input voltage at different solution concentrations.

The influence of solution concentration on NO and NOx removal efficiency is displayed in Figure 7 . The liquid temperature is $20^{\circ} \mathrm{C}$ and the power discharge frequency is $8 \mathrm{kHz}$. It can be seen from Figure 7a that under the same energy density, increasing the solution concentration can improve NO removal efficiency, and this effect is particularly significant when the solution concentration is lower than $0.1 \mathrm{~mol} / \mathrm{L}$. Specifically, when the KCL solution with a concentration of $0.001 \mathrm{~mol} / \mathrm{L}$ is used as the ground electrode, the energy density at the initial discharge reaches $2.2 \mathrm{~kJ} / \mathrm{L}$, and the maximum $\mathrm{NO}$ removal efficiency is only $58 \%$ at the energy density of $4.8 \mathrm{~kJ} / \mathrm{L}$. The NO removal effect is enhanced remarkably as the solution concentration increases to $0.01 \mathrm{~mol} / \mathrm{L}$ and a NO removal efficiency of $100 \%$ is achieved at the energy density of $4.2 \mathrm{~kJ} / \mathrm{L}$. However, the profiles of the $\mathrm{NO}$ removal efficiency versus energy density at the solution concentration of $0.1 \mathrm{~mol} / \mathrm{L}$ and $1.0 \mathrm{~mol} / \mathrm{L}$ are basically the same, and both the NO removal efficiency reach $100 \%$ at the energy density of $3.0 \mathrm{~kJ} / \mathrm{L}$. Considering the relationship between the concentration and conductivity of the KCL solution, the results demonstrate that the influence of solution conductivity on NO removal effect becomes negligible as it reaches $10 \mathrm{~ms} / \mathrm{cm}$ or higher. The variation of NOx removal efficiency with solution concentration is similar to that of $\mathrm{NO}$, as shown in Figure $7 \mathrm{~b}$. At the solution concentration of $0.001 \mathrm{~mol} / \mathrm{L}$, the maximum 
NOx removal efficiency is $28.75 \%$ as the energy density increases to $4.88 \mathrm{~kJ} / \mathrm{L}$. Half of the energy density is required for the same NOx removal efficiency when a KCL solution with a concentration of $0.01 \mathrm{~mol} / \mathrm{L}$ is used. Furthermore, under the same energy density, the NOx removal efficiency is significantly improved with the increase of solution concentration. When the solution concentration is $1.0 \mathrm{~mol} / \mathrm{L}$ and the energy density is $3.15 \mathrm{~kJ} / \mathrm{L}$, the NOx removal efficiency reaches up to $85.24 \%$.
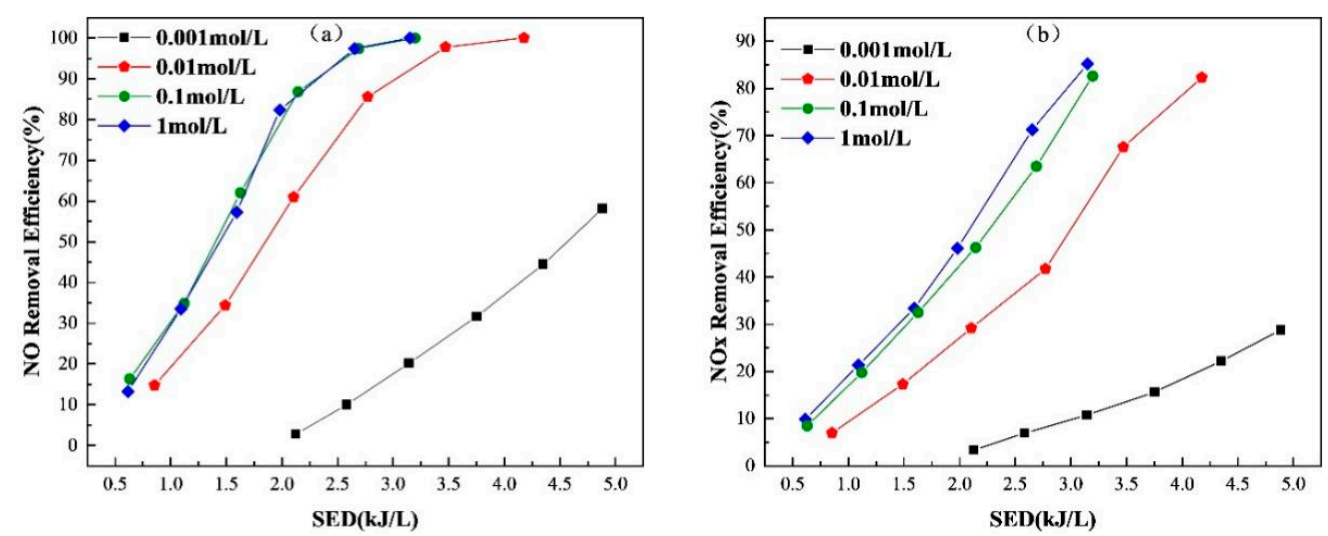

Figure 7. The influence of solution concentration of the ground electrode on NO/NOx removal efficiency at different SEDs: (a) NO removal efficiency; (b) NOx removal efficiency.

\subsection{The Influence of Solution Temperature on NOx Removal Efficiency}

Gas temperature is one of the critical factors affecting the reaction rate of each chemical reaction in the DBD reactor. During the experiment, the temperature of the ground electrode directly determines the temperature of the reaction gas, which in turn affects the NOx removal effect. In order to clarify the influence of gas temperature on NOx removal efficiency, the NOx removal experiments at different solution temperatures are conducted in this part.

The results are exhibited in Figure 8 and the data in the left and right columns are obtained at the KCL concentrations of $0.001 \mathrm{~mol} / \mathrm{L}$ and $0.1 \mathrm{~mol} / \mathrm{L}$, respectively. Figure $8 \mathrm{a}, \mathrm{b}$ depict the variation of the energy density with input voltage at solution temperatures of $30^{\circ}$, $40^{\circ}, 50^{\circ}$, and $60^{\circ}$. When the solution concentration is $0.001 \mathrm{~mol} / \mathrm{L}$, the energy density of the DBD reactor decreases with the increase of solution temperature. For example, the energy density is $4.5 \mathrm{~kJ} / \mathrm{L}$ at the input voltage of $110 \mathrm{~V}$ when the solution temperature is $30^{\circ}$, and a $20 \%$ drop is obtained as the temperature rises to $60^{\circ}$. Therefore, increasing gas temperature can improve the discharge effect of the reactor under such concentration. On the contrary, the difference in energy density between different temperatures is very small when the solution concentration is set to $0.1 \mathrm{~mol} / \mathrm{L}$ (Figure $8 \mathrm{~b}$ ). This shows that after the solution conductivity of the ground electrode reaches a certain value, the gas temperature has little effect on the energy consumption per unit volume of the DBD plasma reactor. The influence of gas temperature on NO removal efficiency at the solution concentrations of $0.001 \mathrm{~mol} / \mathrm{L}$ and $0.1 \mathrm{~mol} / \mathrm{L}$ are shown in Figure $8 \mathrm{c}, \mathrm{d}$. Obviously, when the concentration of the ground electrode is $0.001 \mathrm{~mol} / \mathrm{L}$, the increase in solution temperature can effectively promote the removal of NO, and a higher energy density is required to achieve the same NO removal efficiency at low temperatures. As the solution concentration increases to $0.1 \mathrm{~mol} / \mathrm{L}$, the curves in Figure 8d are basically the same under different temperatures, indicating that the influence of gas temperature on NO removal effect becomes negligible. In particular, a $100 \% \mathrm{NO}$ removal efficiency is achieved when the energy density reaches $2.7 \mathrm{~kJ} / \mathrm{L}$ or higher regardless of the gas temperature. 

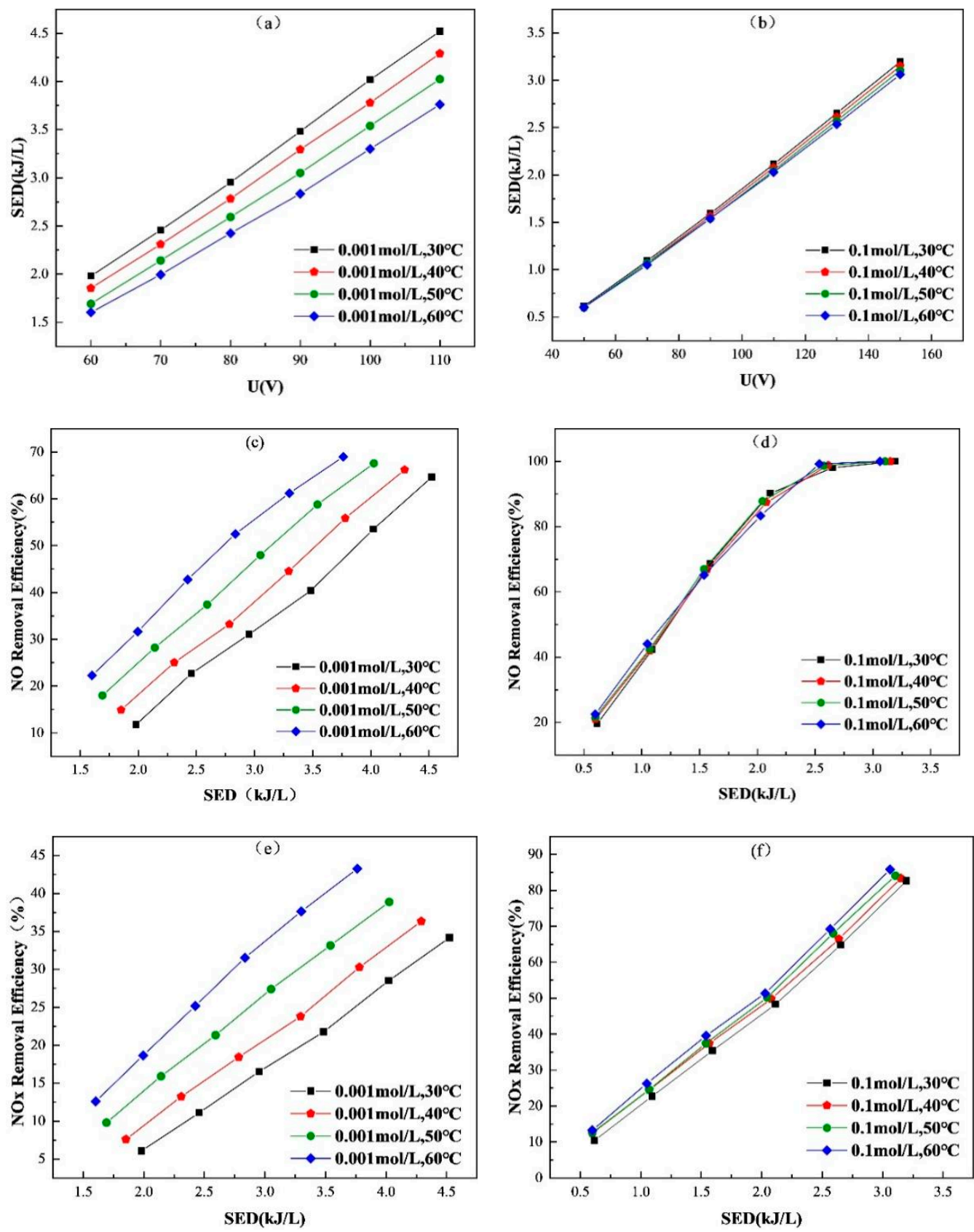

Figure 8. The influence of gas temperature and KCL solution concentration on NO/NOx removal efficiency: (a) The SED changes with input voltage at $0.001 \mathrm{~mol} / \mathrm{L}$; (b) The SED changes with input voltage at $0.1 \mathrm{~mol} / \mathrm{L}$; (c) NO removal efficiency at $0.001 \mathrm{~mol} / \mathrm{L}$; (d) NO removal efficiency at $0.1 \mathrm{~mol} / \mathrm{L}$; (e) NOx removal efficiency at $0.001 \mathrm{~mol} / \mathrm{L}$, and (f) NOx removal efficiency at $0.1 \mathrm{~mol} / \mathrm{L}$.

A similar variation trend is observed as to the influence of gas temperature on NOx removal efficiency. It can be seen from Figure $8 \mathrm{e}$ that the increase in temperature significantly improves the removal of NOx when the solution concentration is $0.001 \mathrm{~mol} / \mathrm{L}$. In fact, corresponding to the solution temperature of $30^{\circ}, 40^{\circ}, 50^{\circ}$, and $60^{\circ}$, the conductivity of the KCL solution is $0.250 \mathrm{~ms} / \mathrm{cm}, 0.253 \mathrm{~ms} / \mathrm{cm}, 0.256 \mathrm{~ms} / \mathrm{cm}$, and $0.260 \mathrm{~ms} / \mathrm{cm}$, respectively, and the absolute change in conductivity is too small to cause such a large increase in NOx removal efficiency. The main reason for this phenomenon is that there is only a layer of $1 \mathrm{~mm}$ thick glass between the reaction gas and the ground electrode, and the NOx removal reaction rate is elevated as the solution temperature increases. The change of NOx removal efficiency with solution temperature at the solution concentration of $0.1 \mathrm{~mol} / \mathrm{L}$ is shown 
in Figure 8f. It is found, by comparison, that the difference in NOx removal efficiency between different temperatures becomes very limited, which further shows that when the conductivity reaches a certain value, the effect of solution temperature on the NOx removal reaction is rapidly reduced. More importantly, the results show the effectiveness and significant advantages of the KCL solution as the ground electrode in NOx removal. When the KCL solution with a concentration of $0.1 \mathrm{~mol} / \mathrm{L}$ is used as the ground electrode, the NOx removal efficiency can reach up to $86 \%$ at the energy density of $3.0 \mathrm{~kJ} / \mathrm{L}$.

\subsection{Spectral Diagnosis}

To better understand the output of the NOx removal experiment, spectral diagnosis is performed at the solution concentration of $1.0 \mathrm{~mol} / \mathrm{L}$. As shown in Figure 9a, when pure nitrogen is introduced into the DBD reactor at the power supply frequency of $8 \mathrm{kHz}$ and the input voltage of $150 \mathrm{~V}$, the spectral line intensity of the second positive band of $N_{2}\left(C^{3} \Pi_{u} \rightarrow B^{3} \Pi_{g}\right)$ molecule is very obvious. Significant peaks are observed at the wavelengths of $316 \mathrm{~nm}, 337 \mathrm{~nm}, 357 \mathrm{~nm}, 375 \mathrm{~nm}$, and $406 \mathrm{~nm}$, which indicates that the nitrogen molecules in the discharge area have undergone a transition from $\mathrm{N}_{2}\left(\mathrm{C}^{3} \Pi_{\mathrm{u}}\right)$ state to $\mathrm{N}_{2}\left(\mathrm{~B}^{3} \Pi_{\mathrm{g}}\right)$ state. In addition, although $99.99 \%$ high-purity nitrogen is used in the experiment, a clear NO- $\gamma$ band appears in the wavelength range of $200 \sim 280 \mathrm{~nm}$. Simek [24] also reported a similar phenomenon, which may be caused by the trace amount of $\mathrm{O}_{2}$ contained in the sample gas. Since the content of $\mathrm{O}_{2}$ is very small, the minute quantity of $\mathrm{NO}$ is mainly generated through Reactions (4)-(6) [20]. Figure $9 \mathrm{~b}$ displays the emission lines of the reactor when $\mathrm{N}_{2}, \mathrm{NO}(500 \mathrm{ppm})$, and $\mathrm{NO}_{2}$ (50 ppm) are introduced. Similarly, there is a distinct second positive band of nitrogen molecules in the wavelength range of $300 \sim 400 \mathrm{~nm}$. Moreover, a high intensity NO- $\gamma$ band in the region of $200 \sim 280 \mathrm{~nm}$ produced by the transition of the excited state $\mathrm{NO}\left(\mathrm{A}_{2} \Sigma^{+}\right)$to the ground state is shown, which demonstrates that $\mathrm{NO}$ is produced during ionization. When $\mathrm{N}_{2}, \mathrm{O}_{2}(5 \%), \mathrm{NO}$ (500 ppm), and $\mathrm{NO}_{2}(50 \mathrm{ppm})$ are filled into the DBD reactor, the emission spectrum of $\mathrm{NO}$ is not observed shown as Figure 9c. This is because a large content of oxygen collides with the high-energy electrons to produce $\mathrm{O}$ atoms in the reactor. The main reactions that may occur in the DBD reactor are Reactions (8)-(10), i.e., $\mathrm{O}_{2}$ in the gas source ionizes to form $\mathrm{O}_{3}$ and reacts with $\mathrm{NO}$ to generate $\mathrm{NO}_{2}$, which reduces $\mathrm{NO}$ [25]. Therefore, when the power supply voltage reaches $150 \mathrm{~V}$ under the $\mathrm{N}_{2}, \mathrm{O}_{2}, \mathrm{NO}$, and $\mathrm{NO}_{2}$ system, the $\mathrm{NO}$ removal efficiency is $100 \%$. Stamate [26] found that when the input power of the plasma reactor is less than $175 \mathrm{~W}$, the ozone in the reactor is mainly consumed by the oxidation of NO to $\mathrm{NO}_{2}$, and $\mathrm{NO}$ decreases sharply as the power increases. When the input power is greater than $175 \mathrm{~W}$, $\mathrm{NO}$ is completely oxidized, and $\mathrm{NO}_{2}$ starts to be slowly oxidized to $\mathrm{N}_{2} \mathrm{O}_{5}$, and the $\mathrm{NO}_{2}$ content decreases. This is consistent with the spectral results of ours. When the input power exceeds a certain value, the main reaction in the reactor is the oxidation of $\mathrm{NO}_{2}$ by $\mathrm{O}_{3}$ to $\mathrm{N}_{2} \mathrm{O}_{5}$. Figure $9 \mathrm{~d}$ shows the variation of spectral intensity with peak discharge voltage in the $\mathrm{N}_{2}, \mathrm{O}_{2}, \mathrm{NO}$, and $\mathrm{NO}_{2}$ system. It can be seen that the spectral intensity is enhanced as the peak discharge voltage increases. The reason for this phenomenon is that when the input voltage increases, both the density of high-energy particles and the energy released by the DBD reactor increase. 

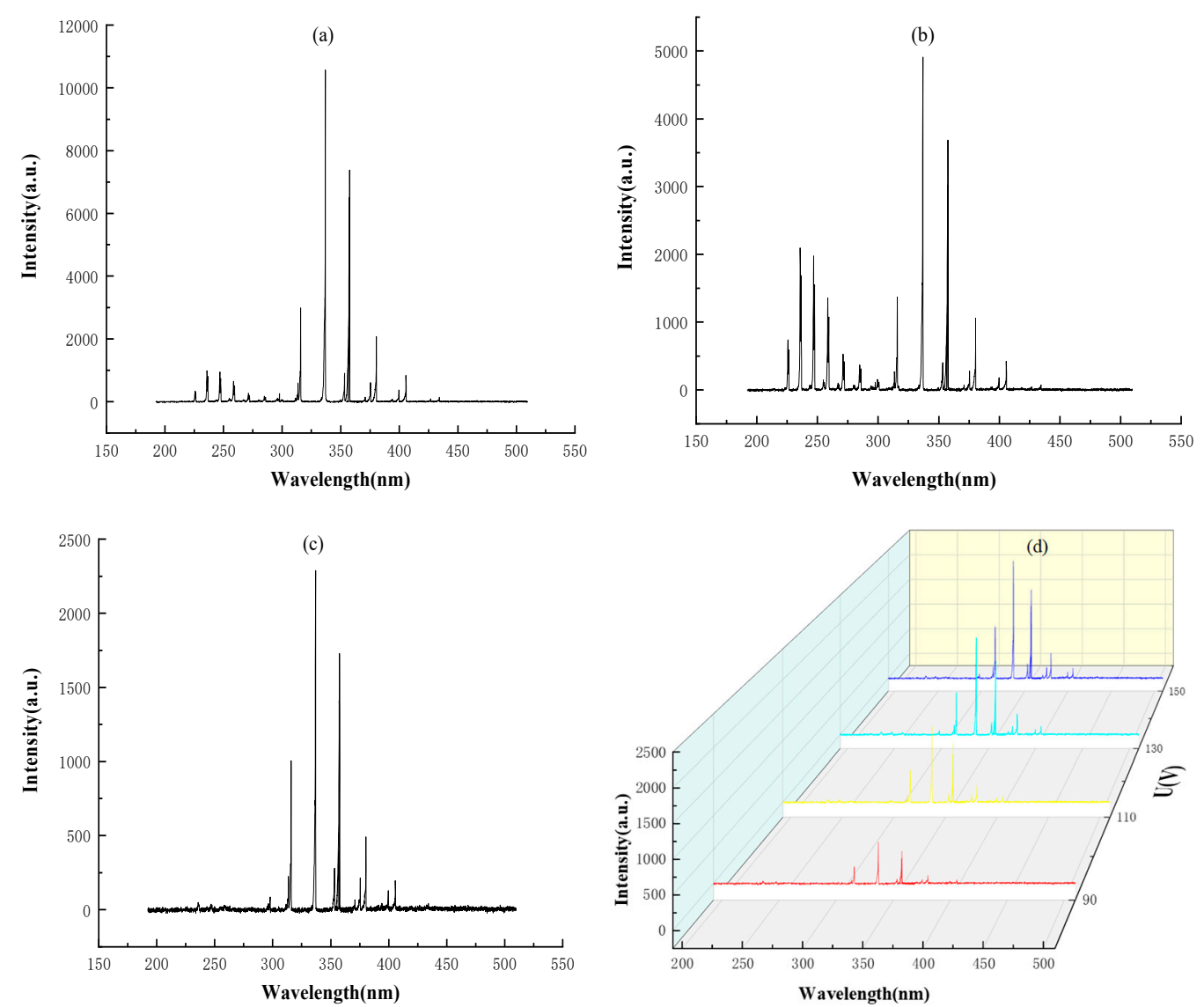

Figure 9. Schematic diagram of emission spectrum distribution: (a) Spectrum of $\mathrm{N}_{2}$; (b) Spectrum of $\mathrm{N}_{2} / \mathrm{NO} / \mathrm{NO}_{2} ;$ (c) Spectrum of $\mathrm{N}_{2} / \mathrm{O}_{2} / \mathrm{NO} / \mathrm{NO}_{2}$, and (d) Spectrum of $\mathrm{N}_{2} / \mathrm{O}_{2} / \mathrm{NO} / \mathrm{NO}_{2}$ at different voltages.

\section{Conclusions}

A series of NOx removal experiments were carried out to study the influence of KCL solution as the ground electrode in a dielectric barrier non-thermal plasma reactor. The results indicate that the use of KCL solution as the ground electrode of the DBD reactor provides a promising strategy to improve the NOx removal efficiency. The conclusions are as follows.

1. The discharge stability and uniformity of the liquid ground electrode are much better than that of the metal mesh electrode.

2. The NOx removal efficiency varies with the power supply frequency. Under the conditions defined in this experiment, the removal effect is the best when the power frequency is $8 \mathrm{kHz}$ and the maximum NOx removal efficiency reaches $85.82 \%$.

3. The NO/NOx removal efficiency increases with the rise of solution conductivity of the ground electrode. Additionally, when the conductivity exceeds $10.00 \mathrm{~ms} / \mathrm{cm}$, the influence of conductivity on NOx removal effect is significantly reduced.

4. When the solution temperature is $30 \sim 60^{\circ} \mathrm{C}$, the NOx removal efficiency increases with the reaction gas temperature, and this phenomenon is particularly significant when the KCL solution concentration is lower than $0.1 \mathrm{~mol} / \mathrm{L}$. A $100 \%$ NO removal efficiency is achieved at the solution concentration of $0.1 \mathrm{~mol} / \mathrm{L}$ regardless of the solution temperature.

5. In the $\mathrm{N}_{2}, \mathrm{NO}$, and $\mathrm{NO}_{2}$ system, the second positive band spectrum of $\mathrm{N}_{2}$ occupies the dominant position. After $\mathrm{O}_{2}$ is added and the power supply voltage is increased to $150 \mathrm{~V}$, the NO spectrum disappears and the NO removal efficiency reaches $100 \%$. 
Author Contributions: Conceptualization, Z.W. and J.Z.; Data curation, Y.G. and W.Z.; Formal analysis, X.X. and W.Z.; Investigation, Y.G. and W.Z.; Resources, Z.W., J.Z. and Y.J.; Software, C.C.; Writing-original draft, X.X. and Y.G.; Writing—review and editing, Z.W. and Y.J. All authors have read and agreed to the published version of the manuscript.

Funding: This research was funded by China Postdoctoral Science Foundation (2021M690496), The Fundamental Research Funds for the Central Universities of China (3132019331), National Natural Science Foundation of China (51876019), and National Key R\&D Program of China (SQ2019YFE011597), Natural Science Foundation of Liaoning Province(2020-HYLH-36).

Institutional Review Board Statement: Not applicable.

Informed Consent Statement: Not applicable.

Data Availability Statement: The data used to support the findings of this study are available from the corresponding author upon request.

Conflicts of Interest: The authors declare no conflict of interest.

\section{References}

1. Zhao, W.X.; Liu, Y.H.C.; Heng, W.; Zhang, R.X. NO Removal by Plasma-Enhanced NH3-SCR Using Methane as an Assistant Reduction Agent at Low Temperature. Appl. Sci. 2019, 9, 2751. [CrossRef]

2. $\quad$ Li, X.; Bei, N.F.; Hu, B.; Wu, J.R.; Pan, Y.P.; Wen, T.X.; Liu, Z.R.; Liu, L.; Wang, R.N.; Li, G.H. Mitigating NOX emissions does not help alleviate wintertime particulate pollution in Beijing-Tianjin-Hebei, China. Environ. Pollut. 2021, 279, 116931. [CrossRef]

3. Kartohardjono, S.; Saksono, N.; Supramono, D.; Prawati, P. NOx Removal from Air through Super Hy-drophobic Hollow Fiber Membrane Contactors. Int. J. Technol. 2019, 10, 472-480. [CrossRef]

4. Wang, Z.Y.; Kuang, H.L.; Zhang, J.F.; Chu, L.L.; Ji, Y.L. Experimental study on the removal of real exhaust pollutants from a diesel engine by activated carbon. Appl. Sci. 2019, 9, 3175. [CrossRef]

5. Nguyen, V.T.; Nguyen, D.B.; Heo, L.; Mok, Y.S. Plasma-Assisted Selective Catalytic Reduction for Low-Temperature Removal of NOx and Soot Simulant. Catalysts 2019, 9, 853. [CrossRef]

6. Dobslaw, D.; Ortlinghaus, O.; Dobslaw, C. A combined process of non-thermal plasma and a low-cost mineral adsorber for VOC removal and odor abatement in emissions of organic waste treatment plants. J. Environ. Chem. Eng. 2018, 6, 2281-2289. [CrossRef]

7. Wang, T.; Sun, B.M. Effect of temperature and relative humidity on NO X removal by dielectric barrier discharge with acetylene. Fuel Process. Technol. 2016, 144, 109-114. [CrossRef]

8. Mei, D.H.; Tu, X. Conversion $\mathrm{CO}_{2}$ in a cylindrical dielectric barrier discharge reactor: Effects of plasma processing parameters and reactor design. J. $\mathrm{CO}_{2}$ Util. 2017, 19, 68-78. [CrossRef]

9. Takaki, T.; Fujiwara, T.; Naito, J.; Sato, T. Improvement of Stability for NO Removal in Multipoint Barrier Discharge Reactor by Pulse Applied Voltage. J. Adv. Oxid. Techonl. 2014, 17, 239-248. [CrossRef]

10. Rehman, F.; Majeed, A.; Abdul, M.; William, B.Z. Hydrogen production from water vapor plasmolysis using DBD-Corona hybrid reactor. Energy Fuels 2013, 27, 2748-2761. [CrossRef]

11. Cai, Y.K.; Lu, L.; LI, P. Study on the Effect of Structure Parameters on NO Oxidation in DBD Reactor under Oxygen-Enriched Condition. Appl. Sci. 2020, 10, 6766. [CrossRef]

12. Zhi, C.; Mathur, V.K. Non-thermal Plasma for Gaseous Pollution Control. Ind. Eng. Chem. Res. 2002, 41, $2081-2089$.

13. Bratislav, B.M.; Obradović, G.B.; Milorda, M.K. A dual-use of DBD plasma for simultaneous NOx and $\mathrm{SO}_{2}$ removal from coal-combustion flue gas. J. Hazard. Mater. 2011, 185, 1280-1286.

14. Yoon, S.; Panov, A.G.; Tonkyn, R.G.; Ebeling, A.C.; Barlow, S.E.; Balmer, M.L. An examination of the role of plasma treatment for lean NOx reduction over sodium zeolite $\mathrm{Y}$ and gamma alumina: Part 1. Plasma assisted $\mathrm{NOx}$ reduction over $\mathrm{Na} \mathrm{Y}$ and $\mathrm{Al}_{2} \mathrm{O}_{3}$. Catal. Today 2002, 72, 243-250. [CrossRef]

15. Cao, X.X. Experimental Study on Removal NOx and VOCs from Flue Gas by Barrier Discharge Plasma Process. Ph.D. Thesis, Shandong University, Jinan, China, 2016.

16. Yang, L.; Zhang, X.; Kan, Q.; Zhao, B.R.; Ma, X.X. Effect of gas composition on nitric oxide removal from simulated flue gas with DBD-NPC method. Chin. J. Chem. Eng. 2019, 27, 3017-3026. [CrossRef]

17. Wang, C.Q.; Zhang, G.X.; Wang, X.X. Comparisons of discharge characteristics of a dielectric barrier discharge with different electrode structures. Vacuum 2012, 86, 960-964. [CrossRef]

18. Chen, Q.Y.; Che, X.K.; Chen, C.; Nie, W.S.; Quan, Y.H.; Zhou, S.Y. Experimental Study on Characteristics of Coaxial Cylinder Dielectric Barrier Discharge with High-frequency AC Actuation. High Volt. Eng. 2020, 46, 3715-3723.

19. Wang, Z.Y.; Kuang, H.L.; Zhang, J.F.; Chu, L.L.; Ji, Y.L. Nitrogen oxide removal by non-thermal plasma for marine diesel engines. RSC Adv. 2019, 9, 5402-5416. [CrossRef]

20. Wang, Z.Y. Study on Catalytic Removal of NOx in Marine Exhaust Gas by Non-Thermal Plasma Assisted Activated Carbon. Ph.D. Thesis, Dalian Maritime University, Dalian, China, 2019. 
21. Bazinette, R.; Sadeghi, N.; Francoise, M. Dual frequency DBD: Influence of the amplitude and the frequency of applied voltages on glow, Townsend and radio frequency DBDs. Plasma Sources Sci. Technol. 2020, 29, 095010. [CrossRef]

22. Liu, B. Research on Removal of NO, by Dielectric Barrier Discharge. Ph.D. Thesis, North China Electric Power University, Beijing, China, 2011.

23. Li, W.J.; Li, X.H.; Cai, Y.X.; Shi, Y.X.; Xu, H. Study on active substance generation and spectral analysis in NTP reactor. Chin. J. Electron. 2017, 40, 819-823.

24. Simek, M.; Babicky, V.; Martin, C. Observation of the $\mathrm{N}_{2}$ Herman infrared system in pulsed positive streamer induced emission at atmospheric pressure. J. Phys. D Appl. Phys. 2001, 34, 3185-3190. [CrossRef]

25. Yang, D.Z. Optical Spectra Characteristic and Application Basic Investigations of Nanosecond Pulsed Dielectric Barrier Discharge at Atmospheric Pressure. Ph.D. Thesis, Dalian University of Technology, Dalian, China, 2012.

26. Stamate, E.; Irimiea, C.; Salewski, M. Investigation of NOx reduction by low temperature oxidation using ozone produced by dielectric barrier discharge. Jpn. J. Appl. Phys. 2013, 52, 05EE03. [CrossRef] 